\title{
Forschung mit Kindern - zur jüngsten Stellungnahme der NEK-CNE
}

\section{Susanne Brauer}

Wissenschaftliche Mitarbeiterin der NEK-CNE

\footnotetext{
* Die Stellungnahme ist in deutscher, französischer, italienischer und englischer Sprache online unter www.nek-cne.ch verfügbar und kann ebenfalls über das Sekretariat der NEK-CNE, c/o BAG, 3003 Bern, bezogen werden.
}

Die Nationale Ethikkommission im Bereich Humanmedizin (NEK-CNE) hat am 11. März 2009 ihre Stellungnahme Nr. 16/2009* veröffentlicht, in der sie sich zu ethischen Fragen rund um Forschung mit Kindern äussert. Die Empfehlungen der Kommission stehen in Zusammenhang mit den gegenwärtigen gesetzgeberischen Arbeiten am Verfassungsartikel und am Bundesgesetz über die Forschung am Menschen. Mit Blick auf die Forschung mit Kindern sieht die Kommission einen besonderen ethischen Klärungsbedarf, der sich aus folgender Ausgangslage ergibt.

Einerseits sind Minderjährige und namentlich Kinder besonders schutzbedürftig, da sie nicht im vollen Sinn urteilsfähig sind und die Entscheidung über die Teilnahme an einer Forschungsstudie ihre gesetzlichen Vertreter (in der Regel die Eltern) treffen. Die Gefahr, Kinder zu instrumentalisieren oder anderweitig nicht in ihrem spezifischen Interesse zu handeln, ist daher potentiell gegeben. Dies gilt speziell im Fall von Forschung, bei der kein möglicher individueller Nutzen für die involvierten Minderjährigen zu erwarten ist (sogenannte «fremdnützige» Forschung).

Andererseits besteht ein grosser Bedarf an Forschung mit Kindern und Jugendlichen, um das medizinische Wissen über sie zu erweitern. Dies betrifft vor allem die Verabreichung und Erprobung von Medikamenten. Gegenwärtig werden Minderjährigen vielfach Präparate verschrieben oder medikamentöse Dosierungen verabreicht, die nicht für die entsprechenden Altersgruppen klinisch geprüft wurden. Kinder sind aufgrund ihrer physiologischen, toxikologischen und pharmakologischen Besonderheiten jedoch keine «kleinen Erwachsenen».

In ihrer Stellungnahme stellt sich die NEKCNE die Aufgabe, ethische Argumente für und wider die Forschung mit Kindern und Jugendlichen auszuloten. In ihren Empfehlungen macht sie sich für eine vermehrte Generierung medizinischen Wissens, das im Interesse der Minderjährigen selbst liegt, stark, fordert hierbei aber die konsequente Einhaltung ethisch gebotener Schutzmassnahmen. Die folgende Darstel- lung gibt einen kurzen Überblick über die wichtigsten Themen und Aussagen der Stellungnahme.

\section{Grundlegende Prinzipien}

In Reaktion auf die menschenverachtenden Forschungspraktiken des Nationalsozialismus in Deutschland wurde 1947 im Nürnberger Kodex die Bedingung aufgestellt, dass Forschung am Menschen nur dann betrieben werden darf, wenn ein Einverständnis der Versuchsperson vorliegt. Diese Bedingung wurde in Folge zum Konzept des «informierten Einverständnisses nach hinreichender Aufklärung» (informed consent) weiterentwickelt und zur zentralen Rechtsfigur für die Legitimation von Forschungshandlungen erhoben. Gerade dieses Konzept ist aber auf Menschen mit fehlender oder eingeschränkter Urteils- und Entscheidungsfähigkeit nicht anwendbar. Um dennoch Forschung an Minderjährigen ermöglichen zu können, deren medizinische Notwendigkeit unbestritten ist, wurden in supranationalen Übereinkommen (z.B. die europäische Biomedizinkonvention) und standesethischen Richtlinien (z.B. die Deklaration von Helsinki des Weltärztebundes) vor allem fünf zentrale Schutzprinzipien formuliert. Zunächst ist nur dann Forschung an Minderjährigen zulässig, wenn deren Erkenntnisse nicht durch Versuche an urteilsfähigen Personen gewonnen werden können (Prinzip der Subsidiarität). Auch muss die Forschungsstudie durch eine unabhängige Ethikkommission bewilligt werden. Für die Teilnahme eines Minderjährigen an einer Studie braucht es als weitere notwendige Voraussetzung das Einverständnis seines gesetzlichen Vertreters. Dies sind in der Regel die Eltern. Unabhängig davon ist jedoch eine Ablehnung des Kindes oder Jugendlichen gegen eine Forschungshandlung zu respektieren. Weiterhin wird für den Fall, dass die Forschungsteilnahme der Versuchsperson keinen möglichen individuellen Nutzen in Aussicht stellt, gefordert, dass das Forschungsprojekt einen Gruppennutzen (z. B. Erweiterung des medizinischen Wissens für eine Altersgruppe, der die Versuchsperson selbst angehört) erwarten lässt und dass die Belastun- 
gen und Risiken für die teilnehmenden Personen minimal sind.

\section{Forschung mit Kindern: die ethische Fragestellung}

In der konkreten Umsetzung dieser rechtlichen Schutzprinzipien können sich ethische Konflikte ergeben. Vielfach gründen diese in der Tatsache, dass Kinder besonders vulnerabel sind, weil sie von Entscheidungen ihrer gesetzlichen Vertreter abhängig, auf deren Fürsorge und Betreuung angewiesen und nur eingeschränkt in der Lage sind, ihre eigenen Interessen zu vertreten und zu schützen. Wegen ihrer seelischen und körperlichen Abhängigkeit sind Kinder nicht immer fähig, ihre Situation zu beeinflussen und sich beispielsweise im Kontext einer Forschungsstudie zu wehren. Einige ausgewählte ethische Fragestellungen, die sich aus dieser Vulnerabilität und besonderen Schutzbedürftigkeit ergeben und in der Stellungnahme der NEK-CNE ausführlich diskutiert werden, sollen im Folgenden aufgegriffen werden.

\section{Schlüsselstelle:}

\section{Beurteilung von Urteilsfähigkeit}

Ausschlaggebend für den Umgang mit Minderjährigen und dem Zugeständnis von Mitsprache- und Abwehrrechten ist die Beurteilung ihrer Urteilsfähigkeit. Diese Fähigkeit wird stets in Bezug auf einen bestimmten Sachverhalt gemessen. So sind die Bedingungen für die Einwilligung einer urteilsfähigen (minderjährigen oder erwachsenen) Person an höhere Standards gebunden als die Abwehr gegen eine Forschungshandlung, die z.B. auch mit nichtsprachlichen Gesten zum Ausdruck gebracht werden kann. Entscheidend bleibt jedoch, von wem und nach welchen Methoden die Urteilsfähigkeit gemessen wird. Hierfür fixe Altersgrenzen zu konstatieren, scheint der NEK-CNE verfehlt, da weniger das Alter als vielmehr die Reife des Kindes bei der Beurteilung ausschlaggebend sein sollte. Emotionale, soziale und motivationale Prozesse sowie Krankheitserfahrungen können die Ausbildung von Urteils- und Einwilligungskompetenz fördern oder beeinträchtigen. Die NEK-CNE geht davon aus, dass sich die kognitiven und voluntativen Kompetenzen des Kindes individuell und graduell entwickeln. Daher wäre für die Beurteilung der kindlichen Reife eine Einzelfallabklärung wünschenswert, um festzulegen, ob allein das stellvertretende Einverständnis oder auch die Zustimmung des Kindes eingeholt werden muss. Diese Feststellung ist gerade in Konfliktfällen entscheidend, in denen beispielsweise die Eltern eine Studienteil- nahme begrüssen, weil sie darin für ihr krankes Kind eine letzte «therapeutische Chance» sehen, das Kind diese Teilnahme aber ablehnt. Im Falle, dass das Kind nach einer kindgerechten Aufklärung kognitiv in der Lage ist, seine Krankheitssituation und das Fehlen von Therapiemöglichkeiten zu verstehen, sollte im Sinne der Stärkung der Persönlichkeitsrechte des Kindes sein Wille ausschlaggebend sein.

\section{Zum Wohl des Kindes - wer bestimmt?}

Die NEK-CNE geht prima facie davon aus, dass Eltern am besten das Wohl und die Interessen des Kindes vertreten können und sich an diesen Grössen in ihren das Kind betreffenden Entscheidungen auch orientieren sollen. Das Wohl beinhaltet dabei sowohl eine objektive (medizinische) Komponente als auch subjektive Elemente, z. B. individuelle Wünsche und Wertvorstellungen. Gerade die subjektiven Elemente sind bei Kindern - und vor allem bei Neugeborenen - noch nicht oder nur in beschränktem Masse ausgebildet. In solchen Fällen muss ein «Wohlverstandenes» objektives Wohl ermittelt werden, das das Entwicklungspotential und die Zukunftsperspektiven des Kindes ebenfalls berücksichtigt. Wenn das Kind sich jedoch äussern kann, dann ist im Konfliktfall mit der elterlichen Definition des Kindeswohls sein Mitspracherecht zu stärken und allenfalls zu überprüfen, ob Eigeninteressen der Eltern ihre Bestimmung dessen, was für das Kind «gut» sei, verzerren.

\section{Messunschärfen: \\ Risiken und Belastungen benennen und gegen Nutzen abwägen}

Dürfen Versuchspersonen in Forschungsprojekten, die ihnen keinen individuellen Nutzen in Aussicht stellen, überhaupt Risiken oder Belastungen ausgesetzt werden? Der Vorentwurf des Humanforschungsgesetzes vom 1. Februar 2006 zum Beispiel schreibt für solche Fälle vor, dass Belastungen und Risiken nur «minimal» sein dürfen. Damit folgt er der etablierten Begrifflichkeit internationaler Regelungen und Richtlinien. Was jedoch in der Praxis als «minimale», d.h. kurzfristige nichtgravierende Beeinträchtigung des Wohlbefindens verstanden wird, ist umstritten. Während beispielsweise einige die Verwendung von Blut für Studienzwecke im Rahmen einer ohnehin vorgenommenen Blutentnahme als minimal einstufen, zählen andere auch die zusätzliche venöse Blutentnahme noch dazu. Auch diagnostische Verfahren wie beispielsweise die Röntgenaufnahme können mit Belastungen einhergehen, die unter Umständen langfristige Folgen haben könnten. 
In den USA wurde versucht, das Bestimmungsproblem mit einem Vergleich zu lösen. Diejenigen Beeinträchtigungen gelten als minimal, die Gefährdungen im Alltag (z.B. Sterberisiko im Strassenverkehr oder Verletzungsgefahr im Sport) entsprechen. Nach Ansicht der NEKCNE ist allerdings eine solche Analogiebildung nicht nur ungenau, sondern auch ethisch problematisch. Sie übersieht die normative Frage, ob es ethisch vertretbar und gesellschaftlich wünschenswert sein sollte, dass Kinder im Alltag solchen z.T. hohen Risiken ausgesetzt sind. Zudem wird in der Analogiebildung übersehen, dass Kinder (auch abhängig von ihrem sozialen Umfeld und Lebensraum) vielen Alltagsrisiken und -belastungen unfreiwillig ausgesetzt sind, während die Teilnahme an einer Studie freiwillig geschieht - und mögliche Gefährdungen in diesem Kontext vermieden werden könnten.

Alternativ empfiehlt die Kommission, die Minimalität von Beeinträchtigungen im Sinne der «Zumutbarkeit» auszulegen (siehe Kasten, 3. Kernaussage). Die Zumutbarkeit leitet sich aus den Dimensionen der Wahrscheinlichkeit und des Schweregrades eines Schadens ab. Entscheidend ist jedoch die subjektive Auslegung der Zumutbarkeit von Beeinträchtigungen, die darauf abhebt, die je spezifische Situation des Kindes (z.B. seinen Entwicklungsstand und seinen Krankheitszustand) wie auch die Art und den Umfang des Nutzens (individuell/gesellschaftlich, gross/gering) mitzuberücksichtigen. Dabei gilt für die NEK-CNE der Grundsatz, dass die Höhe des Risikos bzw. der Belastung im Verhältnis zur Art und zur Höhe des erwartbaren Nutzens stehen muss. Wenn mit der Teilnahme an einer Studie die begründete Hoffnung besteht, dass sich die Lebensqualität des Kindes verbessern kann, ist es vertretbar, höhere Risiken und Belastungen hinzunehmen. Eine analoge Argumentation ist jedoch für Forschungen, aus denen nur Dritte einen Nutzen ziehen, nach Ansicht der NEK-CNE unzulässig. Auch utilitaristische Überlegungen, möglichst vielen Kindern mit der Entwicklung neuer medizinischer diagnostischer, therapeutischer oder präventiver Verfahren zu helfen, können eine unzumutbare Beeinträchtigung des Wohls der Versuchsperson nicht rechtfertigen.

Damit sind wir beim schwierigsten Punkt angelangt: Kann Forschung, die keinen möglichen individuellen Nutzen für die Probandin oder den Probanden beinhaltet, gerechtfertigt werden? Die prinzipielle Wichtigkeit solcher Forschung steht für die NEK-CNE ausser Frage. So braucht man beispielsweise für die Entwicklung neuer diagnostischer Verfahren Untersuchungen von gesunden Kindern, um Normal- und Referenzwerte zu erheben. Kritiker sogenannter «fremdnütziger» Forschung sehen jedoch darin eine Instrumentalisierung von Kindern zu Gunsten Dritter, da die Probandinnen und Probanden selbst keinen Nutzen aus der Forschungsstudie ziehen können. Eine solche Instrumentalisierung stelle eine auch verfassungsrechtlich nicht zulässige Missachtung der Würde des Kindes dar. Befürworter wenden hingegen ein, dass eine Instrumentalisierung hier nur «partiell» geschieht und mit denen im Alltag vergleichbar sei. Eine Studienteilnahme sei daher keine unzulässige Form der Instrumentalisierung; im Gegenteil: man könne es als eine nicht vertretbare Diskriminierung von einwilligungsunfähigen Personen verstehen, wenn diese vom medizinischen Fortschritt ausgeschlossen werden.

\section{Gruppennutzen und Solidarität - was kann man damit rechtfertigen?}

Im Zusammenhang mit sogenannter «fremdnütziger» Forschung prüft die NEK-CNE in ihrer Stellungnahme verschiedene Argumente, die für die Legitimierung von Forschung ohne möglichen individuellen Nutzen im Rahmen der schweizerischen Gesetzgebungsprozesse vorgebracht wurden, namentlich die Verweise auf eine Solidarität mit kranken Kindern und auf einen Gruppennutzen. Während manche bestreiten, dass man Solidarität der Kinder in Anspruch nehmen darf, wenn nur ein Einverständnis der gesetzlichen Stellvertreter vorliegt, weil dies eine Verletzung von Persönlichkeitsrechten repräsentiert («nur ich darf entscheiden, ob ich altruistisch oder egoistisch handeln möchte»), gibt die NEK-CNE zu bedenken, dass bei Kindern durchaus ein Gefühl der Solidarität gegenüber anderen Kindern wahrnehmbar ist. Auch Eltern zeigen oft die Bereitschaft, ihr Kind in «fremdnützige» Studien zu involvieren. Von diesen empirischen Beobachtungen ausgehend, wäre es für die NEK-CNE jedoch unzulässig, eine Pflicht zu solidarischem Handeln zu formulieren oder daraus eine Erziehungsmassnahme zu formulieren. Die Fähigkeit der Kinder zum solidarischen Handeln legt jedoch eine Erlaubnis solcher Forschung nahe.

Grössere Schwierigkeiten bereitet der NEKCNE jedoch das Argument des Gruppennutzens, das als zusätzliche Schutzmassnahme beispielsweise im Vorentwurf des Humanforschungsgesetzes eingeführt wurde. Hier scheinen mehrere Aspekte problematisch. Zunächst lässt sich nicht klar definieren, was eine «Gruppe» ist. Ordnungen nach Altersspannen, Krankheitstypen, aber auch nach sozialen, ökonomischen und geogra- 
phischen Kriterien wären hier denkbar. Schwerer wiegt jedoch der Einwand, dass damit eine Forschung an Kindern für Erwachsenenkrankheiten nicht zulässig wäre. Gerade aber solche Studien könnten im Interesse des Kindes liegen, da es selbst einmal erwachsen wird. Zudem ist nicht einsichtig, warum das Kind sich nur mit einer bestimmten Gruppe solidarisch zeigen soll, anstatt mit allen Menschen. Angesichts dessen, dass die Gruppendefinition von Dritten vorgenommen und damit eine Form der Fremdbestimmung ist, können Bedenken aufkommen, dass hier eine «Schicksalsgemeinschaft» konstruiert wird, die nicht dem realen Empfinden der Betroffenen entspricht. Auch der argumentative Versuch, den Gruppennutzen als einen «erweiterten Eigennutzen» zu interpretieren, ist für die NEK-CNE nicht überzeugend; denn für das individuelle Kind bleibt der Gruppenutzen immer ein Fremdnutzen.

Ein alternativer Vorschlag führt ein «objektives Interesse» des Kindes an Forschung ins Feld, das darin besteht, dass angesichts der Möglichkeit des Kindes, selbst zu erkranken, dem Kind ein Interesse unterstellt werden darf, dass es die Erforschung solcher Krankheiten sowie einen medizinischen Fortschritt im allgemeinen begrüsst. Bei dem «objektiven Interesse» handelt es sich um eine Hypothese, die durchaus widerlegt werden kann, z.B. wenn das Kind Zeichen der Abwehr gegen Forschungshandlungen zeigt.

\section{Vier Kernaussagen der NEK-CNE zu Forschung mit Kindern}

1. Es gibt einen ethisch begründeten Bedarf für Forschung mit Kindern.

2. «Therapeutische» Forschung mit Kindern muss ein angemessenes Verhältnis von Nutzen und Belastungen, von Chancen und Risiken für das betroffene Kind selbst aufweisen.

3. Forschung mit Kindern ohne möglichen individuellen Nutzen ist aus ethischen Gründen nicht auszuschliessen, erfordert aber äusserste Vorsicht. Die Formulierung, dass Risiken und Belastungen in Forschungsvorhaben ohne möglichen individuellen Nutzen nur «minimal» sein dürfen, soll im Sinne der «Zumutbarkeit» für die Kinder ausgelegt werden.

4. Nicht jede Anwendung von Medikamenten bei Kindern verlangt zwingend eine klinische Studie. Auch langjährige therapeutische Erfahrung in der Praxis rechtfertigt deren Anwendung.
In Anbetracht dieser Versuche, Forschung ohne möglichen individuellen Nutzen für das Kind auf eine ethisch vertretbare Grundlage zu stellen, kommt die NEK-CNE zu dem Schluss, dass für den Schutz des Kindes folgende Kriterien ausschlaggebend sind: die Achtung des Subsidiaritätsprinzips, die Einwilligung des gesetzlichen Vertreters bzw. urteilsfähigen Minderjährigen, sowie die Zumutbarkeit von Risiken und Belastungen. Als zusätzliche Schutzkriterien wären zu empfehlen, dass das Abwehrrecht der Kinder an niedrigere Bedingungen geknüpft und die Studie begleitend kontrolliert wird, z. B. durch den Einsatz einer externen Vertrauens- und Ansprechperson. Ein Verweis auf einen Gruppennutzen, ein Solidaritätsgefühl oder ein «objektives Interesse» zählt hingegen nicht zu den notwendigen Kriterien für die Zulassung sogenannter «fremdnütziger» Studien, auch wenn der Wert solcher Studien unter Umständen damit aufgezeigt werden könnte.

\section{Empfehlungen der NEK-CNE}

In ihren Empfehlungen (siehe Kasten) macht sich die NEK-CNE für eine vermehrte Generierung medizinischen Wissens, das im Interesse der Minderjährigen selbst liegt, stark, fordert hierbei aber die konsequente Einhaltung ethisch gebotener Schutzmassnahmen. Zu diesen Schutzmassnahmen gehören unter anderem, nur Forschung mit Kindern zuzulassen, die nicht mit Urteilsfähigen durchgeführt werden könnte, das Kind über die Studie kindgerecht aufzuklären und Zeichen seiner Ablehnung gegen Forschungshandlungen gebührendes Gewicht zu geben. Zudem sollte «therapeutische» Forschung mit Kindern ein angemessenes Verhältnis von Nutzen und Belastungen sowie von Chancen und Risiken für das betroffene Kind aufweisen. Wo Forschung für die Versuchsperson selbst keinen potentiellen Nutzen bringt, müssen Risiken und Belastungen für das Kind «zumutbar» sein. Ebenfalls ist es der NEK-CNE wichtig zu betonen, dass nicht jede Anwendung von Medikamenten bei Kindern zwingend eine klinische Studie verlangt, sondern auch langjährige therapeutische Erfahrung eine Verabreichung rechtfertigen kann. 